\title{
EFFECT OF AMMONIUM CERIC NITRATE AS INITIATOR IN GRAFTING OF ACRYLIC ACID ONTO PINE CONE POWDER
}

\author{
NOZIPHO NOMPUMELELO MZINYANE, HARRY CHIRIRIWA, \\ AUGUSTINE ENAKPODIA OFOMAJA and ELIAZER BOBBY NAIDOO \\ Biosorption and Water Research Laboratory, Department of Chemistry, \\ Vaal University of Technology, P. Bag X021, Vanderbiljpark 1900 South Africa \\ \Corresponding author: Nozipho Mzinyane,noziphom@vut.ac.za
}

\begin{abstract}
Chemical grafting of acrylic acid onto pine cone powder was carried out using free radical initiation. Pretreatment by Fenton oxidation was used for the removal of extractives, such as plant pigments, lignin, resin acids and tannins, for the accessibility of cellulose to ceric ammonium nitrate initiator and to the monomer. The effects of temperature, monomer (acrylic acid), initiator (CAN), time, acid $\left(\mathrm{HNO}_{3}\right)$ and mass of pine powder were investigated. The optimum conditions were found to be $70{ }^{\circ} \mathrm{C}$, monomer to mass of pine ratio of $7: 10,1 \mathrm{~g}$ of initiator, $1 \mathrm{~g}$ of Fenton pine cone powder, 0.9 $\mathrm{mol} / \mathrm{L} \mathrm{HNO}_{3}$ and reaction time of 60 minutes. Acetone and tetrahydrofuran (THF) were used to remove the homopolymer (poly(acrylic acid)) from the grafted copolymer. A relatively high percentage of grafting was achieved under optimum reaction conditions. Fenton pretreatment of the pine cone powder before grafting provided the formation of the hydroxyl radical, which improved the grafting percentage and the efficiency to $100 \%$ and $54 \%$, respectively. Furthermore, the advantages of using CAN provided high grafting efficiency and lower homopolymer formation. The grafted copolymer was characterized by FTIR, TGA, DSC, SEM, XRD and EDS analyses.
\end{abstract}

Keywords: grafting, acrylic acid, ceric ammonium nitrate, Fenton reagent, pine cone powder

\section{INTRODUCTION}

Green chemistry has generated renewed interest in novel composites derived from a variety of agricultural waste materials. ${ }^{1}$ Agricultural by-products are available in large quantities and have potential as inexpensive sorbents. These natural materials are eco-friendly due to their unique chemical composition. ${ }^{2}$ Adsorbents resulting from agricultural waste materials are usually called biosorbents. ${ }^{3}$

Pine cone is a lignocellulose material, it is an abundant natural resource from the wood industry and large quantities are produced annually. It belongs to the class of Gymnosperma and family of Pinaceae, and is a renewable resource. ${ }^{2}$ Pine plantations are grown for the furniture, building and paper industries and the cones are available as a waste product. Nurseries grind the cones into mulch or they are sold as crafts, but the consumer demand for these cones is small, compared to the by-products from other industries. ${ }^{4}$

Ridout et al..$^{5}$ reported that lignocellulosic biomass is composed mainly of cellulose $(40 \%)$, hemicelluloses (20\%), and lignin (18\%). ${ }^{6,7}$ Hence, chemical or biological treatment to prevent the leaching of colored organic compounds, such as tannins, and to depolymerize others, such as lignin, is necessary. ${ }^{4}$ Different modification techniques, which include the use of inorganic acids (sulphuric acid, nitric acid, phosphoric acid, hydrochloric acid, citric acid, tartaric acid), strong bases (calcium hydroxide, sodium hydroxide, sodium carbonate), oxidising agents (Fenton's reagent, potassium permanganate), organic compounds (ethylenediaminetetraacetic acid, formaldehyde, epichlorohydrin), grafting and solvent extraction have been reported in the literature by several authors. ${ }^{2,-10}$

Ofomaja et $a l^{6}$ reported that chemical treatment with potassium hydroxide $(\mathrm{KOH})$ solubilized a small fraction of the lignin as well as soluble extracts in the plant waste, and improved the penetration of modifying agents. They further reported that chemical grafting using radical initiators is known to form covalent linkages between biosorbent and polymer molecules, which are not reversible under low solution $\mathrm{pH}$ conditions. Grafting using different monomers onto cellulosic or lignocellulosic materials has been used to enhance their chemical and physical properties by allowing the attachment of a wide 
range of specific groups to the cellulose backbone. $^{11}$

Grafting acrylonitrile onto pineapple leaf fibers improved their thermal properties, grafting acrylamide onto coconut husk produced a selective adsorbent for mercury (II), while grafting acrylamide onto sisal fibers improved their moisture absorption. ${ }^{10}$ To improve the structural characteristics of the initial material, a pretreatment is necessary for removing plant organic components, such as plant pigments, lignin, resin acids and tannins for enhancing the accessibility of cellulose to the initiator and to the monomer. ${ }^{12}$ Bhange et al. ${ }^{13}$ reported an increased grafting percentage when the cellulose fiber was pretreated by passing nitrogen through the aqueous solution, which was agitated in the Fenton reagent initiator. ${ }^{13}$ Hence, the type of pretreatment and initiator has an important effect on the grafting and it determines the grafting percentage depending on the monomer to be grafted. $^{12}$

The pretreatment by the Fenton reagent (Iron (II) hydrogen peroxide) is cheap, because it involves the addition of hydrogen peroxide to a solution containing lignocellulose material in the presence of ferrous salts, creating hydroxyl radicals. ${ }^{6}$ Bhange et al. ${ }^{14}$ investigated the effect of Fenton's pretreatment on garden biomass lignin and cellulose degradation, and their results showed that Fenton's reagent was effective in lignin degradation.

Additionally, the type of initiator has an important effect on grafting, for example, ceric ion offers many advantages due to its high grafting efficiency and lower homopolymer formation. Radical formation on the cellulose backbone may occur either on the carbon (C-6) or oxygen atom of methylol $\left(-\mathrm{CH}_{2} \mathrm{OH}\right)$ group. ${ }^{10,11}$ Therefore, this study seeks (1) to pretreat the surface of pine cone powder by using Fenton's reagent and (2) to graft with acrylic acid using ceric ammonium nitrate as initiator. The resulting composite will be then characterized using FTIR, TGA, DSC, SEM, XRD and EDS.

\section{EXPERIMENTAL}

\section{Sample collection}

The cones were collected from pine trees (Gymnosperma class, Pinaceae family) in Vanderbijlpark, Gauteng province in South Africa. Megasporophylls (female pine cones), i.e. cone scales, were used for this study. They were washed and dried in an oven at $80{ }^{\circ} \mathrm{C}$ for 48 hours to remove impurities and some volatile organics, such as monoterpenes, without destroying the pine cone matrix. The cone scales were peeled and crushed to powder using a pulveriser, and then sieved. The fraction with particle size ranging between $45-90 \mu \mathrm{m}$ was retained for experimental use.

\section{Reagents}

All the reagents were purchased from SigmaAldrich or Merck and were used without further purification: $\mathrm{H}_{2} \mathrm{O}_{2}(30 \%), \mathrm{FeCl}_{2} \cdot 4 \mathrm{H}_{2} \mathrm{O}(98 \%), \mathrm{HCl}$ (32\%), $\mathrm{HNO}_{3}(70 \%)$, ammonium ceric nitrate $(98.5 \%)$, acrylic acid (99\%), acetone $(99.5 \%)$ and tetrahydrofuran $(99.5 \%)$.

\section{Physical measurements}

Infrared spectra $\left(500-4000 \mathrm{~cm}^{-1}\right)$ were recorded on a Perkin Elmer FTIR/FT-NIR spectrometer. TGA and DSC were performed using a Thermal Analyzer (STA) 6000. The chemical composition and morphology were investigated by a Scanning Electron Microscope (SEM/EDS) at different $\mathrm{KV}$ magnifications, using a VEGA 3 TESCAN model. Particle size distribution was measured using the laser diffraction technique (Malvern Mastersizer 2000 Instrument).

\section{Synthesis}

The sieved pine cone powder was then divided into two parts. The first part was left untreated and the second was modified with Fenton's reagent and then grafted with acrylic acid.

\section{Fenton's reagent}

Fenton's reagent was prepared according to the procedure described by Ofomaja et al. ${ }^{8}$ The procedure includes measuring $303 \mathrm{~mL}$ of $30 \% \mathrm{H}_{2} \mathrm{O}_{2}$ and $6.993 \mathrm{~g}$ of $\mathrm{Fe}^{2+}$ as $\mathrm{FeCl}_{2} \cdot 4 \mathrm{H}_{2} \mathrm{O}$, which were separately placed in $1000 \mathrm{~mL}$ flasks containing distilled water. The $\mathrm{pH}$ of the $\mathrm{Fe}^{2+}$ solution was adjusted to be between 3 and 4.5 with $0.1 \mathrm{M} \mathrm{HCl}$. An Adwa AD8000 pH meter was used for the $\mathrm{pH}$ measurements. In a $500 \mathrm{~mL}$ threenecked round-bottomed flask with a nitrogen inlet and magnetic stirrer, $250 \mathrm{~mL}$ of $\mathrm{Fe}^{2+}$ and $50 \mathrm{~g}$ of pine cone powder were mixed and heated at $50{ }^{\circ} \mathrm{C}$ for 30 minutes. To this mixture, $250 \mathrm{~mL}$ of the prepared $\mathrm{H}_{2} \mathrm{O}_{2}$ was added and the heating was continued for another 30 minutes. The resulting bleached/oxidised mixture was filtered under suction with a Whatman TM filter paper. The residue was washed with distilled water until the filtrate was clear, and then dried overnight at $80{ }^{\circ} \mathrm{C}$.

\section{Grafting}

Table 1 shows the values of the materials and conditions used for the grafting procedure. Fentontreated pine cone powder was mixed with $20 \mathrm{~mL}$ of distilled water, then nitric acid was added and the mixture was placed in the reaction setup. Ceric ammonium nitrate (CAN) was dissolved in distilled 
water and then added to the mixture. The mixture was heated at the required temperature under vacuum for 5 minutes, and then switched to nitrogen gas for 15 minutes. The required ratio of acrylic acid was added to the mixture gradually over 15 minutes (Table 1), with continuous stirring. The mixture was then centrifuged and the solid was washed with distilled water to remove traces of initiator and acid, dried at room temperature for 24 hours and then washed again with acetone $(2 \mathrm{x} \quad 30 \mathrm{~mL})$ and $(2 \mathrm{x} \quad 30 \mathrm{~mL})$ tetrahydrofuran (THF) to remove the homopolymer (poly(acrylic acid)). The grafting percentage and efficiency for the composite were calculated using Equations 1 and 2.

Grafting percentage $\left.(\%)=\left[\left(\mathrm{W}_{1}-\mathrm{W}_{0}\right) / \mathrm{W}_{0}\right)\right] \times 100$

where $\mathrm{W}_{1}$ and $\mathrm{W}_{0}$ are the weights of the grafted copolymer and the original Fenton treated pine cone powder (FPCP), respectively. ${ }^{10}$

Grafting efficiency (GE) shows the fraction of monomer grafted onto FPCP from the amount of monomer converted to graft polymer plus the remaining homopolymer, which means the fraction of polymer that is grafted to FPCP in total polymer, and it was calculated by using Equation 2.
Grafting efficiency $(\%)=\left[\left(\mathrm{W}_{1}-\mathrm{W}_{0}\right) /\left(\mathrm{W}_{1}-\mathrm{W}_{0}+\mathrm{W}_{2}\right)\right] \mathrm{x}$ 100

where $\mathrm{W}_{1}, \mathrm{~W}_{0}$ and $\mathrm{W}_{2}$ are the weights of the grafted copolymer, the original FPCP, and the homopolymer, respectively. ${ }^{10}$

\section{RESULTS AND DISCUSSION Fourier transform infrared spectroscopy (FTIR) analysis}

Figure 1 shows the infrared spectra for untreated pine cone powder, FPCP and grafted copolymer. The major peak at $3300 \mathrm{~cm}^{-1}$ corresponds to the $-\mathrm{OH}$ band and that at $2900 \mathrm{~cm}^{-1}$ to the $\mathrm{C}-\mathrm{H}$ band; the main difference lies in their intensities. The $-\mathrm{OH}$ band for FPCP declined relative to that of the untreated pine cone powder, which indicated the removal of hydroxyl groups during the Fenton treatment. ${ }^{9,10}$ The increase in intensity of the $-\mathrm{OH}$ band for the grafted copolymer is evidence that grafting with acrylic acid was achieved. ${ }^{6}$

Table 1

Variables used in the grafting procedure

\begin{tabular}{lc}
\hline Variable & Value \\
\hline Mass of pine $(\mathrm{g})$ & $1.0,1.5,2.0,2.5,3.0$ \\
Mass of CAN $(\mathrm{g})$ & $1.0,1.5,2.0,2.5,3.0$ \\
Temperature $\left({ }^{\circ} \mathrm{C}\right)$ & $30,40,50,60,70,80$ \\
Time $($ minutes $)$ & $15,30,45,60$ \\
{$\left[\mathrm{HNO}_{3}\right](\mathrm{M})$} & $0.3,0.6,0.9,1.1$ \\
Acrylic acid ratios & $2: 10,3: 10,4: 10,5: 10,6: 10,7: 10,8: 10,9: 10,10: 10,11: 10,12: 10,13: 10,14: 10$ \\
\hline
\end{tabular}

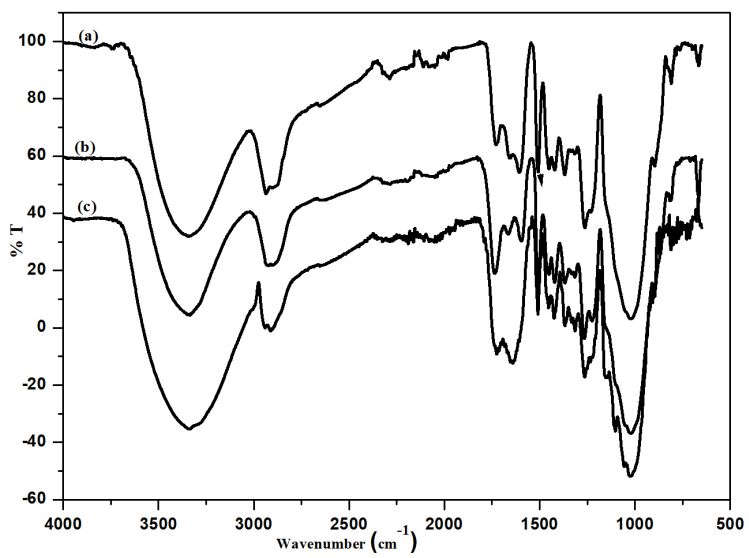

Figure 1: Infrared spectra of (a) untreated pine cone, (b) Fenton treated pine cone and (c) grafted pine cone

The $\mathrm{C}=\mathrm{O}$ band at $1750 \mathrm{~cm}^{-1}$ indicates the asymmetric and symmetric stretching vibrations of the ionic carboxylic acid group. The grafted copolymer signal increased in intensity, indicating that the carboxylic functions had been grafted onto the surface of the FPCP material. The weakening 
of the peak at $1263 \mathrm{~cm}^{-1}$ of the grafted copolymer is due to the deformation vibration of $\mathrm{C}=\mathrm{O}$ and stretching formation of $\mathrm{OH}$ for carboxylic acid. The peak between 2750 and $2900 \mathrm{~cm}^{-1}$, which is attributed to aliphatic $\mathrm{C}-\mathrm{H}$ stretching, decreased for both FPCP and grafted copolymer. This may have been caused by the aliphatic fraction of waxes, which decreased during the Fenton treatment. ${ }^{9}$ The peak at $1500 \mathrm{~cm}^{-1}$ is due to the benzene ring from lignin. ${ }^{6,9,14}$ The lignin fingerprint region (1830-730 $\left.\mathrm{cm}^{-1}\right)$ might be rich in methoxyl $-\mathrm{O}-\mathrm{CH}, \mathrm{C}-\mathrm{O}-\mathrm{C}$ stretching and $\mathrm{C}=\mathrm{C}$ (aromatic ring) containing compounds. ${ }^{15,16}$

FTIR has revealed the functional groups that were present in the three materials (such as hydroxyl, carboxylic, methoxyl and benzene groups). The band intensity of the $-\mathrm{OH}$ group for the grafted copolymer increased compared to the result for FPCP, which shows that the oxidizing agent did oxidize some functional groups on the lignocellulose material and that radical grafting was achieved. ${ }^{17}$

The decrease in intensity of the $\mathrm{C}-\mathrm{H}$ absorption band for FPCP may be attributed to the reduction of the hydrocarbon content, which resulted from the reaction of the starting material (untreated pine cone powder) with the Fenton reagent and grafting with acrylic acid. On the other hand, the weakening of the $\mathrm{C}-\mathrm{O}-\mathrm{C}$ band for the grafted copolymer confirms that cellulose is degraded during grafting. ${ }^{18}$

Fenton's treatment resulted in the oxidation of organic components in carbonaceous materials by hydroxyl radicals generated from the reaction of hydrogen peroxide. The radicals created were transferred to the carbonaceous material (RH), thus breaking down the organic matrix and producing radicals $\left(\mathrm{R}^{\circ}\right)$, which can be oxidized to breakdown, as described in Equations 3 and $4:{ }^{19}$

$$
\begin{aligned}
& \mathrm{Fe}^{2+}+\mathrm{H}_{2} \mathrm{O}_{2} \longrightarrow \mathrm{Fe}^{3+}+\mathrm{OH}^{\circ} \\
& \mathrm{RH}+\mathrm{HO}^{\circ} \longrightarrow \mathrm{H}_{2} \mathrm{O}+\mathrm{R}^{\circ}
\end{aligned}
$$

Furthermore, the cerium ammonium nitrate (CAN) created the initiation sites for grafting on the cellulose backbone. The radical formation on the cellulose backbone may occur either on the carbon (C-6) or on the oxygen atom of methylol $\left(-\mathrm{CH}_{2} \mathrm{OH}\right)$ group and the grafting may also occur on $\mathrm{C}-2$ carbon by the ring opening of the cellulose backbone. Sharma et al. ${ }^{20}$ also reported that grafting occurs mainly at the $\mathrm{C} 2-\mathrm{C} 3$ glycol unit, and to a lesser degree at the C6-hydroxyl in the grafting of acrylonitrile onto Cassia tora gum, which is a common herbaceous annual weed growing in India.

\section{Thermogravimetric (TGA) analysis}

TGA was performed to determine the mass changes in the composite, which happen at a molecular level, as a function of time and temperature. Thermal degradation of the pine cone biomass occurred in three phases: (1) moisture evaporation in the range of $30-150{ }^{\circ} \mathrm{C}$, (2) hemicelluloses and cellulose decomposition at 200-350 ${ }^{\circ} \mathrm{C}$, and (3) lignin decomposition at 160$700{ }^{\circ} \mathrm{C}$, as shown in Figure 2 (a) and (b). ${ }^{21}$ Mass losses are observed in the temperature range of 200-375 ${ }^{\circ} \mathrm{C}$ for untreated powder, while for the grafted copolymer - at $450-750{ }^{\circ} \mathrm{C}$.

Figure 2 (a) shows the first phase corresponding to the initial mass loss at low temperatures below $150{ }^{\circ} \mathrm{C}$ for the untreated pine cone powder and FPCP, which can be explained by water molecules evaporating from the material due to the hygroscopic nature of the pine cone surface. ${ }^{6,22}$ The grafted copolymer showed an initial weight loss at about $150{ }^{\circ} \mathrm{C}$, which is slightly different from that of untreated pine cone powder and FPCP. Mass losses during this stage were of about $10 \%$ for the untreated pinecone powder, $7 \%$ for FPCP and $8 \%$ for the grafted copolymer. The difference in mass losses indicates a decrease in surface hydrophilic groups, which signifies an increase in the hydrophobic character for FPCP and grafted copolymer due to the $\mathrm{Fe}^{2+} / \mathrm{H}_{2} \mathrm{O}_{2}$ treatment and acrylic acid grafting. This information supports the results obtained from the FTIR spectra in Figure 1, which show a loss of hydroxyl functions for both FPCP and grafted copolymer. The second phase (Fig. 2 (a)) shows the decomposition of hemicelluloses at $100-200{ }^{\circ} \mathrm{C}$ and that of cellulose at $200-300{ }^{\circ} \mathrm{C}$ for both untreated pine cone powder and FPCP. The behaviour of the grafted copolymer was slightly different: the decomposition of hemicelluloses occurred at $300-450{ }^{\circ} \mathrm{C}$, while cellulose decomposed at about $450-700{ }^{\circ} \mathrm{C}$. The mass losses were of about 55\% and 50\% for the untreated pine cone powder and FPCP, respectively. However, the mass loss for the grafted copolymer was of about $60 \%$. It was reported that hemicelluloses decompose at lower temperatures owing to their random and amorphous structure. ${ }^{22}$ Cellulose consists of both amorphous and crystalline regions, with the amorphous regions being oxidized at a lower temperature, whereas the crystalline regions are 
oxidized at a higher temperature. The third phase (Fig. 2 (a)) is attributed to the decomposition of lignin and it took place at about $100-700{ }^{\circ} \mathrm{C}$ for the untreated pine cone powder and FPCP, while for the grafted copolymer - at about $200-700{ }^{\circ} \mathrm{C}$. Lignin is chemically different from hemicelluloses and cellulose, because it is composed of three kinds of benzene-propane units, is heavily cross-linked and has a relatively high molecular weight. ${ }^{23,24}$
Additionally, the thermal stability of lignin is also relatively high. The shift that was observed for the grafted copolymer may be attributed to the grafting of acrylic acid onto FPCP, which improved the thermal stability of the grafted copolymer. Enhanced thermal and physicochemical properties of cellulose pine needles grafted with butyl acrylate were also observed. ${ }^{25}$
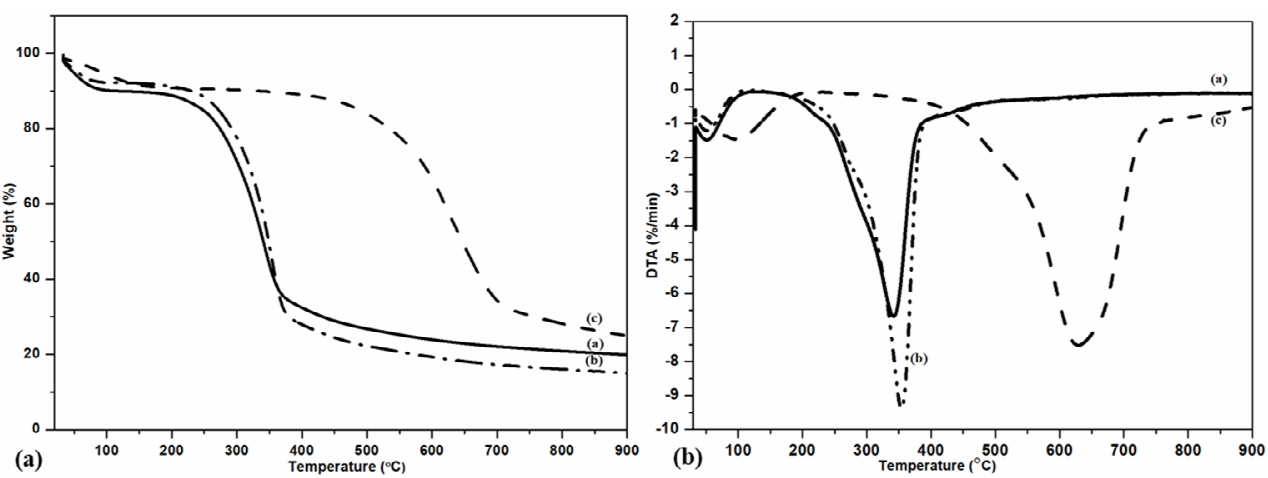

Figure 2: (A) TGA for (a) untreated pine cone, (b) Fenton treated pine cone and (c) grafted pine cone; (B) DTA for (a) untreated pine cone, (b) Fenton treated pine cone and (c) grafted pine cone

The grafted copolymer showed improved thermal stability by its both TGA and DTA curves. The initial decomposition temperature for the grafted copolymer increased from 250 to 450 ${ }^{\circ} \mathrm{C}$. It was observed that $\mathrm{T}_{\max }$ shifted to $750{ }^{\circ} \mathrm{C}$ for the grated pine cone powder, as seen in Figure 2 (b), compared to the decomposition temperature of $350{ }^{\circ} \mathrm{C}$ for untreated pine cone powder and FPCP. These observations clearly indicate that grafting improved the thermal stability of FPCP. Thermal stability was found to be dependent on the degree of grafting onto pine. ${ }^{26}$

\section{X-ray diffraction (XRD) analysis}

XRD was used to determine the crystallographic structure of the studied materials. The corresponding spectra are presented in Figure 3. Compared to the spectrum of untreated pine cone powder, those of FPCP and grafted copolymer show a slight increase in intensity and broadening of the peaks at $2 \theta=11^{\circ}, 15.3^{\circ}, 22.6^{\circ}$ and $34.5^{\circ}$. The spectra reveal that pine cone is composed mainly of crystalline cellulose, as indicated by the peak at $2 \theta=22.6^{\circ} .{ }^{23}$ The peaks at
$2 \theta=15^{\circ}$ and $34.5^{\circ}$ intensified because the crystalline nature of FPCP and grafted copolymer increased. The further increase of the peak at $2 \theta=$ $34.5^{\circ}$ in Figure 3 (c) may be explained by the fact that the amorphous phase of the grafted copolymer was further enhanced by the initiator (CAN), creating more reaction sites on the cellulose backbone as a result of the incorporation of acrylic acid. ${ }^{27}$

\section{Scanning electron microscopy (SEM) analysis}

The surface features of the three studied materials were examined by SEM, and their corresponding micrographs are illustrated in Figure 4 . The surface of the untreated pine cone powder was uneven, with visible small particles, which may be due to the lignin and other extractives. FPCP shows less surface roughness and the small particles are absent, which may be because of the removal of lignin and other extractives, such as soluble organic substances. ${ }^{28}$ The smooth surface of the grafted copolymer can be an indication that acrylic acid coated the surface of FPCP. 


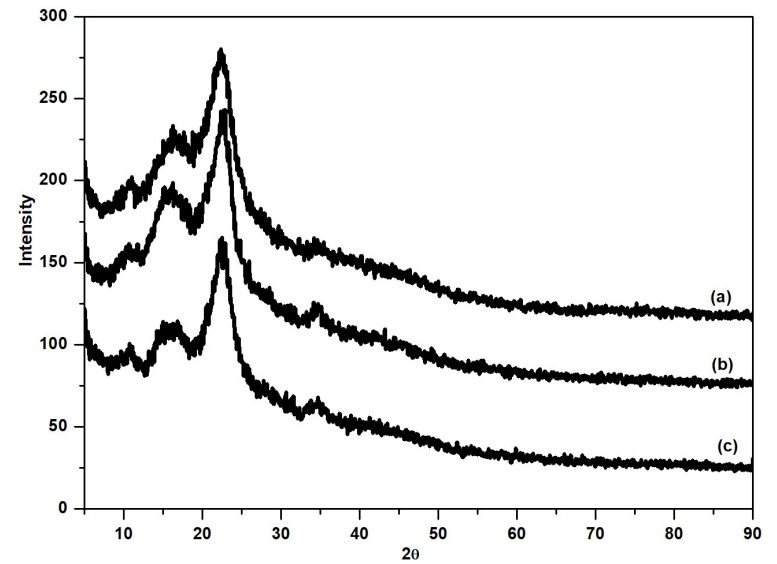

Figure 3: XRD spectra of (a) untreated pine cone, (b) Fenton treated pine cone and (c) grafted pine cone
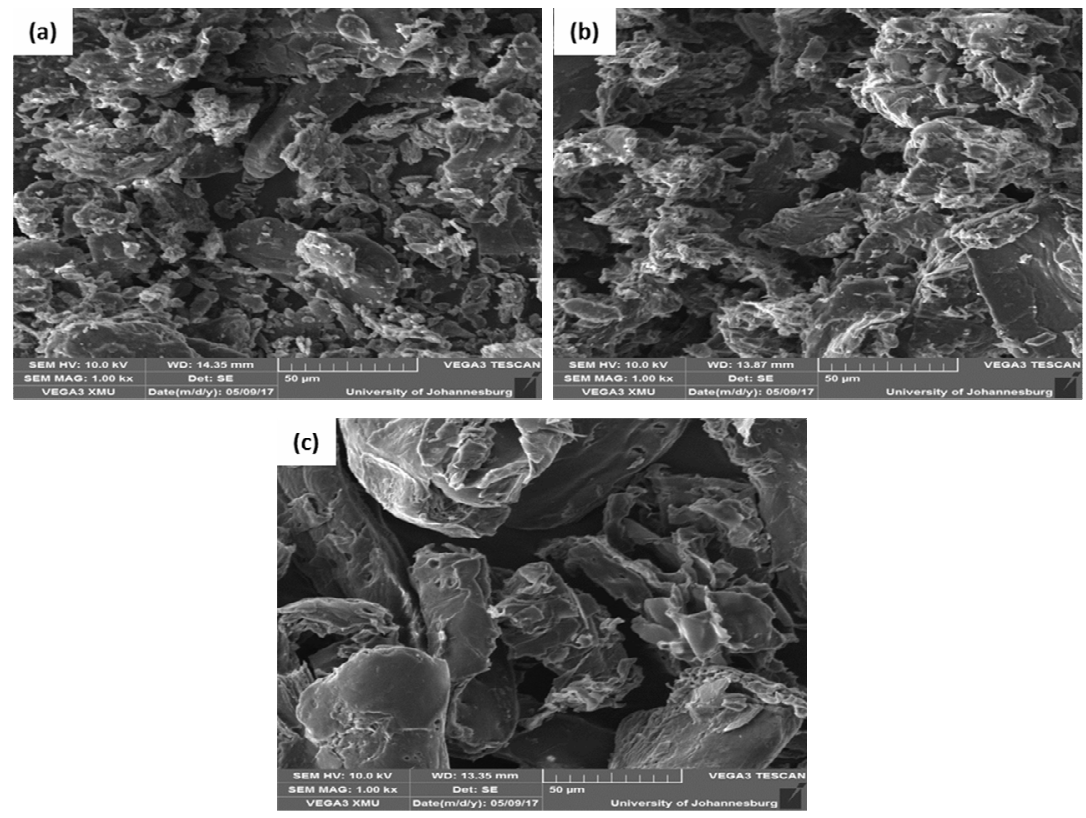

Figure 4: SEM photographs of (a) untreated powder, (b) FPCP and (c) grafted copolymer

\section{Energy dispersive spectroscopy (EDS) analysis}

Table 2 shows the EDS analysis that was performed on untreated pine cone powder, FPCP and grafted copolymer. The amount of carbon in Fenton treated pine and grafted copolymer increased in comparison with that in the untreated pine cone powder. A slight increase in the oxygen content was observed compared to both untreated pine cone powder and Fenton treated pine. This signifies that the carboxylic functional groups had been grafted onto the surface of the FPCP material, as indicated by the FTIR results. The main difference between the untreated pine cone, Fenton treated and the grafted copolymer is in the nitrogen content. This reveals that Fenton's reagent managed to remove some of the extractives, waxes and resin from the Fenton treated pine cone powder. ${ }^{29}$ The highest percent increase in nitrogen content was observed for the grafted copolymer. This signifies that grafting took place on Fenton treated pine powder.

\section{Grafting optimization}

The optimum conditions for copolymerization were investigated in terms of FPCP mass, time, $\mathrm{HNO}_{3}$ concentration, mass of CAN, temperature and monomer ratio, individually, while keeping all the other reaction conditions constant (e.g. time -30 minutes, $\mathrm{HNO}_{3}$ concentration $-0.9 \mathrm{M}$, mass of initiator $-1.0 \mathrm{~g}$, temperature $-50{ }^{\circ} \mathrm{C}$, and 
monomer ratio $-2: 10)$. The corresponding results with respect to grafting percentage and efficiency are shown in Figure 6.

\section{Effect of FPCP mass}

The effects of the FPCP mass were studied and the copolymerization was carried out with various masses ranging from $1.0 \mathrm{~g}$ to $3.0 \mathrm{~g}$ (Fig. 6 (a)). Figure 6 (a) reveals that when the mass of FPCP was $1.0 \mathrm{~g}$, the highest grafting percentage and grafting efficiency were of $92 \%$ and $50 \%$, respectively. A further increase in the amount of FPCP showed a decrease in both parameters. This may be because at $1.0 \mathrm{~g}$ of FPCP, free radicals are generated for grafting the monomer. A further increase in the quantity of FPCP was therefore pointless, because the amounts of acid, initiator and monomer were reduced and the grafting had stopped, since there were no more active sites available.

\section{Effect of time}

There was a slight increase in grafting percentage and efficiency with an increase in time (Fig. 6 (b)), the optimum values achieved being of $99 \%$ and $50 \%$, respectively, at 60 minutes. The increasing grafting percentage with time could be explained by the increased interactions between the initiator, the monomer and FPCP, which resulted in further generation of free radical sites. $^{27}$

\section{Effect of nitric acid concentration}

The optimum grafting percentage and grafting efficiency of $100 \%$ and $51 \%$, respectively, were achieved when the concentration of nitric acid was $0.9 \mathrm{M}$ (Fig. 6 (c)). A further increase in acid concentration showed a slight decrease in grafting percentage, but grafting efficiency remained at $51 \%$.

\section{Effect of initiator (CAN)}

Grafting percentage (99\%) and efficiency $(50 \%)$ were the highest when $1.0 \mathrm{~g}$ of initiator was used (Fig. 6 (d)). A further increase from 1.5 to $3 \mathrm{~g}$ in the mass of initiator led to a slight decrease. This might be attributed to the fact that only initiator concentration was increased, while the concentration of nitric acid, the mass of FPCP and of the monomer remained constant. Nitric acid in the grafting medium allows the initiator to create free radicals on FPCP by oxidizing $\mathrm{Ce}^{4+}$ ions. Therefore, a further increase in the amount of initiator, while the concentration of nitric acid remains constant, resulted in an excess, because the monomer was exhausted and could no longer be attached to free radicals. The initial increase in grafting percentage and efficiency was attributed to the availability of more free radicals generated for grafting the monomer. Maximum yields were observed when an initiator concentration of $1.0 \mathrm{~g}$ and $10 \mathrm{~mL}$ of $\mathrm{HNO}_{3}$ were used. ${ }^{30}$

\section{Effect of monomer}

Grafting parameters started to fluctuate when the concentration of nitric acid was $0.8 \mathrm{M}$. The highest optimum grafting percentage of $100 \%$ and efficiency of $54 \%$ were observed when the monomer ratio was 7:10 (Fig. 6 (e)). This may be explained by assuming that, initially, the active sites of FPCP were available for the monomer, resulting with an increase in grafting percentage and efficiency. However, a further increase in monomer concentration caused more homopolymer to be formed, leading to a decrease in the grafting parameters, because there was excess monomer and limited availability of FPCP.

\section{Effect of temperature}

The maximum grafting percentage of $100 \%$ was obtained at $70{ }^{\circ} \mathrm{C}$, while grafting efficiency was $51 \%$. The grafting parameters started decreasing with a further rise in temperature. These results indicate that the grafting parameters depend on temperature. It was reported that, at 70 ${ }^{\circ} \mathrm{C}$, a higher dissociation rate, diffusion of the initiator and mobility of the monomer to the active sites of cellulose produced a considerable improvement in grafting parameters. ${ }^{31} \mathrm{~A}$ further increase in temperature beyond $70{ }^{\circ} \mathrm{C}$ resulted in radical termination, which led to the decrease of grafting percentage and efficiency. It was observed that at temperatures above $70{ }^{\circ} \mathrm{C}$, more homopolymer was formed and the grafting reaction decreased. Moreover, the optimum temperature for the grafting reaction was observed to be dependent on the initiator used. ${ }^{32}$ Grafting acrylic acid onto cellulose in a heterogeneous medium using $\mathrm{CAN}-\mathrm{HNO}_{3}$ initiator resulted in higher grafting percentages at temperatures between 30 and $70{ }^{\circ} \mathrm{C}$, but lower at $90{ }^{\circ} \mathrm{C} .{ }^{10}$ 
Table 2

Elemental analysis of pine cone (\% on dry weight basis).

\begin{tabular}{lccccc}
\hline Sample & Carbon & Hydrogen & Nitrogen & Sulphur & Oxygen* \\
\hline Untreated pine & 45.37 & 6.381 & 0.33 & 0.108 & 47.81 \\
Fenton treated pine & 46.03 & 6.301 & 0.18 & 0.007 & 47.48 \\
Grafted pine & 44.09 & 6.078 & 0.11 & 0.022 & 49.70 \\
\hline
\end{tabular}

*Calculated by difference
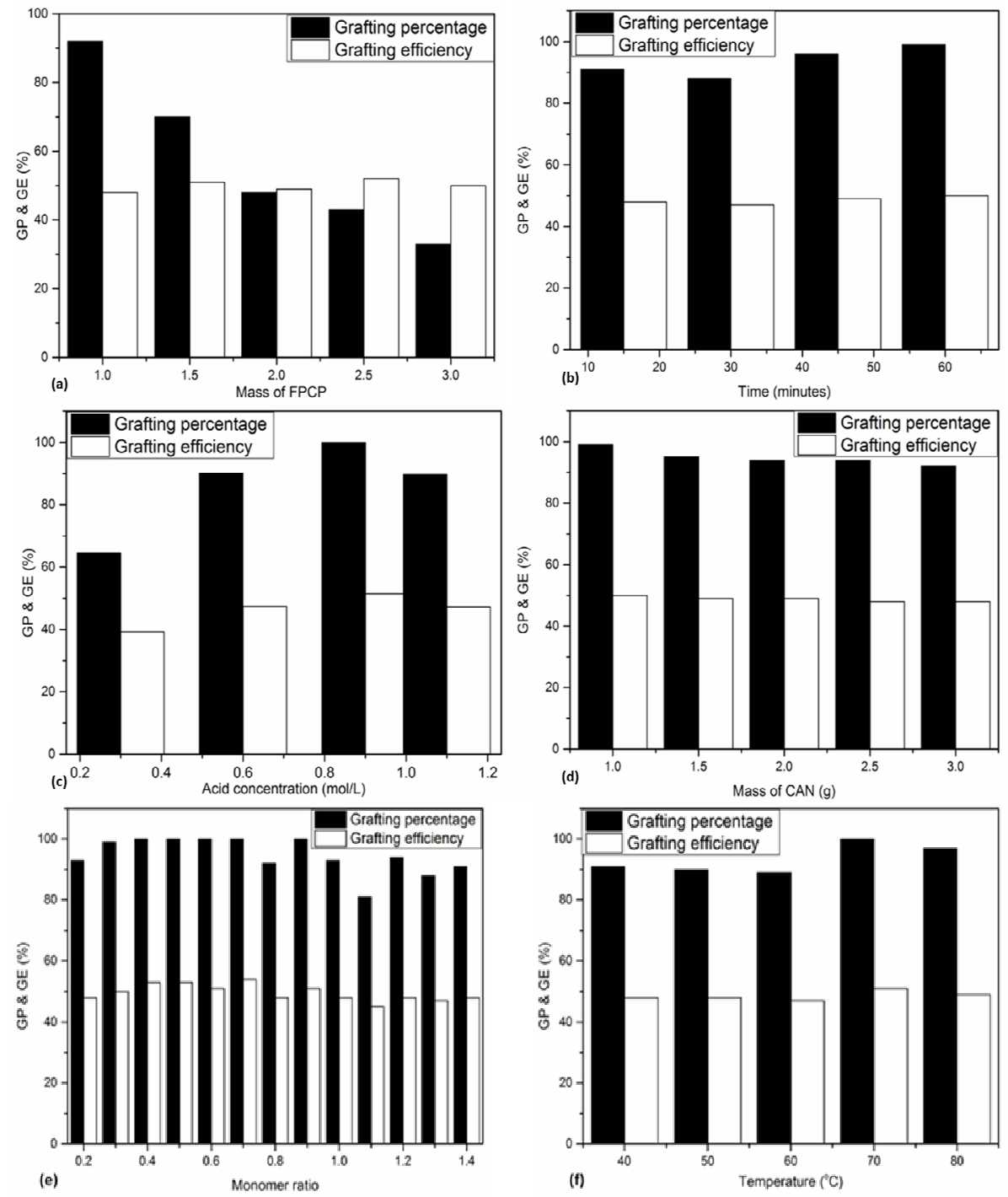

Figure 6: Grafting percentage and efficiency as a function of (a) mass of FPCP, (b) time, (c) acid concentration, (d) mass of CAN, (e) monomer ratio (AA) and (f) temperature

\section{CONCLUSION}

Grafting of acrylic acid onto Fenton treated pine cone powder was successfully conducted by using the free-radical grafting procedure. The optimum conditions for the graft polymerization were established to be: $70{ }^{\circ} \mathrm{C}$, monomer to pine mass ratio $-7: 10$, initiator $-1.0 \mathrm{~g}$, Fenton pine cone powder $-1.0 \mathrm{~g}, \mathrm{HNO}_{3}-0.9 \mathrm{~mol} / \mathrm{L}$ and time of 60 minutes. The highest grafting percentage and efficiency of $100 \%$ and $54 \%$, respectively, were achieved under the optimum reaction conditions. The results obtained from the FTIR, 
TGA, DTA, XRD, EDS and SEM analyses confirmed the presence of acrylic acid on the grafted product. The morphological and structural changes observed showed that grafting was definitely achieved because the crystallinity of the grafted copolymer increased in comparison with that of the untreated pine cone powder and FPCP.

\section{REFERENCES}

1 P. Jain and N. Vigneshwaran, Bioresour. Technol., 103, 219

(2012), https://doi.org/10.1016/j.biortech.2011.09.110

2 A. E. Ofomaja, E. B. Naidoo and S. J. Modise, Desalin. Water Treat., 19, 275 (2010), https://doi.org/10.5004/dwt.2010.1230

3 A. Pholosi, A. E. Ofomaja and E. B. Naidoo, J. Saudi Chem., 17, 77 (2013), https://doi.org/10.1016/j.jscs.2011.10.017

4 A. M. C. J. J. Ridout, PhD Dissertation, Stellenbosch University, March 2016, pp. 12, http://www.sun.ac.za

5 M. D. Teli and J. Sheikh, Cellulose Chem. Technol., 46, 53 (2012).

6 A. E. Ofomaja, E. B. Naidoo and S. J. Modise, J. Environ. Manage., 91, $1674 \quad$ (2010), https://doi.org/10.1016/j.jenvman.2010.03.005

7 M. Madhavi, M. B. Ahmad, Md. J. Haron, M. Z. Ab. Rahman and A. Fatehi, BioResources, 6, 5110 (2011),

https://bioresources.cnr.ncsu.edu/BioRes_06/BioRes_0 6_4_5110_Mahdavi_AHRF_Optim_Graft_copol_PA M_Rubberwd_Fiber_1967.pdf

8 A. E. Ofomaja, S. L. Ngema and E. B. Naidoo, Carbohyd. Polym., 90, $201 \quad$ (2012), https://doi.org/10.1016/j.carbpol.2012.05.024

9 F. A. Abu-Ilaiwi, M. B. Ahmad and N. A. Ibrahim, Polym. Int., 53, $386 \quad$ (2004), https://doi.org/10.1002/pi.1355

10 G. Gürdag and S. Sarmad, in "Polysaccharide Based Graft Copolymers", edited by S. Kalia and M. W. Sabaa, Springer-Verlag, Berlin Heidelberg, 2013, Chapter 2, pp. 15-57, https://doi.org/10.1007/978-3642-36566-9_2

11 S. Hokkanen, A. Bhatnagar and M. Sillanpää, Water Res., 91, $156 \quad$ (2016), https://doi.org/10.1016/j.watres.2016.01.008

12 A. Neira, M. Tarraga and R. Catalan, J. Chil. Chem. Soc., 53, (2008), http://dx.doi.org/10.4067/S0717-97072008000100016

13 V. P. Bhange, S. P. M. Prince William, A. Sharma, J. Gabhane, A. N. Vaidya et al., J. Environ. Health Sci., 13, 12 (2015), https://doi.org/10.1186/s40201015-0167-1

14 P. Garside and P. Wyeth, Stud. Conserv., 48, 269 (2003), https://doi.org/10.1179/sic.2003.48.4.26

15 H. Yang, R. Yan, H. Chen, D. H. Lee and C. Zheng, Fuel, 86, 1781 (2007), https://doi.org/10.1016/j.fuel.2006.12.013
16 M. Cheng, H. He, H. Zhu, W. Guo, W. Chen et al., Carbohyd. Polym., 203, $246 \quad$ (2019), https://doi.org/10.1016/j.carbpol.2018.09.051

17 Y. He, Y. Pang and Y. Liu, X. Li and K. Wang, Energ. Fuels., 22, $2775 \quad$ (2008), https://doi.org/10.1021/ef8000967

18 A. N. Nada, M. Y. Alkady and H. M. Fekry, BioResources, 3, $46 \quad$ (2007), https://bioresources.cnr.ncsu.edu/BioRes_03/BioRes_0 3_1_0046_Nada_AF_SynthChar_GraftedCellulose_M etal.pdf

19 A. J. K. Kupeta, E. B. Naidoo and A. E. Ofomaja, J. Clean. Prod., 179, $191 \quad$ (2018), https://doi.org/10.1016/j.jclepro.2018.01.034

20 B. R. Sharma, V. Kumar and P. L. Soni, J. Appl. $\begin{array}{llll}\text { Polym. } & \text { Sci., } & \text { 90, } & 129\end{array}$ https://doi.org/10.1002/app.12593

21 N. Gao, A. Li and C. Quana, J. Anal. Appl. Pyrol., 100, 26

(2013),

https://doi.org/10.1016/j.jaap.2012.11.009

22 M. Carrier, A. Loppinet-Serani, D. Denux, J.-M. Lasnier, F. Ham-Pichavant et al., Biomass Bioenerg., 35, $298 \quad$ (2011), https://doi.org/10.1016/j.biombioe.2010.08.067

${ }_{23}$ M. Poletto, H. L. Ornaghi Júnior and A. J. Zattera, Materials, $\quad 7, \quad 6105 \quad$ (2014), https://doi.org/10.3390/ma7096105

24 S. S. Idris, N. A. Rahman and K. Ismail, Bioresour. Technol., $\quad \mathbf{1 0 1}, \quad 4584 \quad$ (2010), https://doi.org/10.1016/j.biortech.2010.01.059

25 V. K. Thakur, "Cellulose-Based Graft Copolymers. Structure and Chemistry", edited by V. K. Thakur, Taylor and Francis Group, Boca Raton, 2015, pp. 165, https://taylorandfrancis.com/

26 K. C. Gupta and K. Khandekar, J. Appl. Polym. $\begin{array}{llll}\text { Sci., } & \text { 86, } & 2631 & \text { (2002), }\end{array}$ https://doi.org/10.1002/app.11448

27 Z. Wan, Z. Xiong, H. Ren, Y. Huang, H. Liu et al., Carbohyd. Polym., 83, $264 \quad$ (2011), https://doi.org/10.1016/j.carbpol.2010.07.048

28 J. A. Micales, J. S. Han, J. L. Davis and R. A. Young, in "Biodeterioration Research", vol. 4, edited by G. C. Llewellyn, W. V. Dashek and C. E. O'Rear, Springer, Boston, 1994, pp. 317-332, https://doi.org/10.1007/978-1-4757-9450-2_25

${ }^{29}$ A. J. K. Kupeta, PhD Thesis, Vaal University of Technology, 2014 , http://digiresearch.vut.ac.za/handle/10352/340

30 A. Shanmugapriya, R. Ramya, M. Venkatachalam and P. Sudha, J. Water Res. Protect., 3, 380 (2011), https://doi.org/10.4236/jwarp.2011.36048

31 R. Khullar, V. K. Varshney, S. Nathani and P. L. Soni, eXPRESS Polym. Lett., 2, 12 (2008), https://doi.org/10.3144/expresspolymlett.2008.3

32 J. R. Witono, I. W. Noordegraf, H. J. Heeres and L. P. B. M. Janssen, Carbohyd. Polym., 90, 1522 (2012), https://doi.org/10.1016/j.carbpol.2012.07.024 